\title{
Effectiveness of the Health Complex Model in Iranian primary health care reform: the study protocol
}

This article was published in the following Dove Press journal:

Patient Preference and Adherence

5 October 2016

Number of times this article has been viewed

\author{
Jafar Sadegh Tabrizi' \\ Mostafa Farahbakhsh ${ }^{2}$ \\ Homayoun Sadeghi- \\ Bazargani ${ }^{3,4}$ \\ Roya Hassanzadeh ${ }^{5}$ \\ Akram Zakeri ${ }^{6}$ \\ Leili Abedi $^{7}$
}

'Tabriz Health Services Management Research Center, Tabriz University of Medical Sciences, Tabriz, Iran; ${ }^{2}$ Research Center of Psychiatry \& Behavioral Sciences, Tabriz University of Medical Sciences, Tabriz, Iran; ${ }^{3}$ Road Traffic Injury Research Center, Department of Statistics \& Epidemiology, Tabriz University of Medical Sciences, Tabriz, Iran; ${ }^{4} \mathrm{WHO}$ Collaborating Center on Community Safety Promotion, Department of Public Health Sciences, Karolinska Institute, Stockholm, Sweden; ${ }^{5}$ Department of Health Services Management, Iranian Center of Excellence in Health Management, School of Management and Medical Informatics, ${ }^{6}$ National Public Health Management Center, Tabriz University of Medical Sciences, Tabriz, 'Department of Statistics and Epidemiology, Faculty of Health, Kerman University of Medical Sciences, Kerman, Iran

Correspondence: Homayoun SadeghiBazargani

Road Traffic Injury Research Center, Department of Statistics \& Epidemiology, Tabriz University of Medical Sciences, Sadeghi Street, Golshahr Square, El Goli Avenue, Tabriz 5I678463 I I, Iran Tel +989144027218

Email homayoun.sadeghi@gmail.com
Background: Iranian traditional primary health care (PHC) system, although proven to be successful in some areas in rural populations, suffers major pitfalls in providing PHC services in urban areas especially the slum urban areas. The new government of Iran announced a health reform movement including the health reform in PHC system of Iran. The Health Complex Model (HCM) was chosen as the preferred health reform model for this purpose.

Methods: This paper aims to report a detailed research protocol for the assessment of the effectiveness of the HCM in Iran. An adaptive controlled design is being used in this research. The study is planned to measure multiple endpoints at the baseline and 2 years after the intervention. The assessments will be done both in a population covered by the HCM, as intervention area, and in control populations covered by the traditional health care system as the control area.

Discussion: Assessing the effectiveness of the HCM, as the Iranian PHC reform initiative, could help health system policy makers for future decisions on its continuation or modification.

Trial registration: The study protocol is registered in Iranian Registry of Clinical Trials under the unique code IRCT201402112150N3.

Keywords: health reform, health complex model, public private partnership, primary health care, health network, capitation, Iran

\section{Introduction}

The main mission of a health care system is to promote health and respond to the needs of people and society in the field of health and diseases. Needs change constantly under economic, social, political, and environmental conditions, and the health care system must adapt to the needs arising from such a change. Health care reform is an opportunity to identify new requirements, fill the gaps created by changes, and inject necessary powers to the health system in order to promote it toward its main mission. ${ }^{1}$

Four factors are considered as driving force for the reform in the health sector: increasing costs of health care, growing expectations of the people, limited resources, and mistrust of the people on the governments due to poor governance, corruption, and inefficiency. ${ }^{2}$ In most low- and middle-income countries (LMICs), primary health care (PHC) is provided by the public sector. Because of limited resources, high costs, and low quality of services, politicians and health policy makers are forced to make changes or replace a new system to meet the people's needs and improve the satisfaction of service providers. ${ }^{3}$

In 1975, in cooperation with the World Health Organization (WHO), Iran started the health worker training project in West Azerbaijan Province, and in 1977, the government adopted PHC as the health service development policy, ${ }^{4,5}$ such that primary health 
care was provided to the target groups in the rural population by health workers, specific health care officers, and physicians in health houses and rural health centers. In the urban population, health services were provided by urban health centers and urban health posts by trained health care staff of dedicated programs and practitioners, giving rise to a success even at the early years of implementation of the system. ${ }^{6-8}$ Details of current Iranian health system are provided in earlier publications by Shadpour and Malekafzali. ${ }^{6,8}$

Despite being successful in some areas, studies show that there are three major gaps in the health care system between public health and medical services, between general medical services and specialty services, and between the health system and other systems. ${ }^{1}$ Some primarily successful projects have been done in the field of health care reform. One of these projects is the public-private partnership. ${ }^{2}$ The participation of the public sector and the private sector aims at improving people's health by combining innovative methods with skills and various resources of multiple organizations. This participation is not an alternative for responsibilities of any organization, but the public sector should stick to its original duties such as support of research, preparation of standards, ensuring service delivery, ensuring high-quality services, improving people's access to services, and creating a vibrant and creative environment in service delivery. ${ }^{3}$ Another project was implemented in 1997 in East Azerbaijan Province of Iran through a public-private partnership model called health cooperatives. These health cooperatives covered 9,000-17,000 people and offered PHC services such as vaccination, mother and child care, family planning, environmental health, and outpatient therapeutic services. Service delivery depended on a specific service package and was financed by the government, which was paid to health centers in a capitation contract. ${ }^{9}$ The Health Cooperative Project seemed to be a successful experience in the field of health system reform, but it was suspended because of changes in governmental policy. After this experience, the only health care delivery system in Iran has been the traditional Iranian health network until recent application of the new PHC reform models. The traditional system suffered some shortcomings including low accessibility in urban areas, availability of skilled human resources, lack of efficient evaluation system, and inadequate expected quality of the provided health care.

One of the main priorities of the current government has been reform of the health system in both areas of treatment and PHC services. ${ }^{10}$ One of the main strategies in the reform of PHC is Health Complex Model (HCM). The main characteristics of the HCM that make it different from the traditional system could be notified as taking advantage of the private sector in health care delivery, application of the more efficient payment methods than the traditional salary system, and decentralization of various administrative and supervisory activities.

One reason behind the requirement for research-based evaluations of health reform activities is to provide the advantages and disadvantages of the newly administered health reform programs in order to provide appropriate and valid information for the policy makers to help in their decision-makings.

As performing studies without research evaluation always questions the research effectiveness and as a result, the reliability of interventions is influenced by effectiveness; hence, these interventions must be evaluated quite scientifically using appropriate methodologies. This paper aims to provide a detailed research protocol for the assessment of the effectiveness of HCM through an appropriate scientific methodology. The main hypotheses of the research include assessing effectiveness of the intervention on some health system performance indicators including health care utilization and responsiveness; community empowerment, $\mathrm{PHC}$, trust and national specific health care program indicators such as noncommunicable diseases control programs and pregnancy and child care programs over an assessment time of 2 years.

\section{Methods}

An adaptive controlled design is being used in this research. The study is planned to measure multiple endpoints at the baseline and 2 years after the intervention. The assessments will be done both in a population covered by the HCM, as intervention area, and in control populations covered by the traditional health care system, as the control area.

The research is being conducted in East Azerbaijan Province with a total population of approximately 3,500,000 people located in northwest of Iran. The HCM is officially started in East Azerbaijan as the pioneer province for implementing the health reform at PHC level.

The health complex project is initially implemented in Iran in one slum area in Tabriz Metropolitan as well as in one district with three counties, both located in East Azerbaijan Province of Iran. However, the government is planning to extend the program to more regions in other districts and provinces of Iran.

A parallel interventional study protocol is developed (Figure 1). The intervention group includes the health 


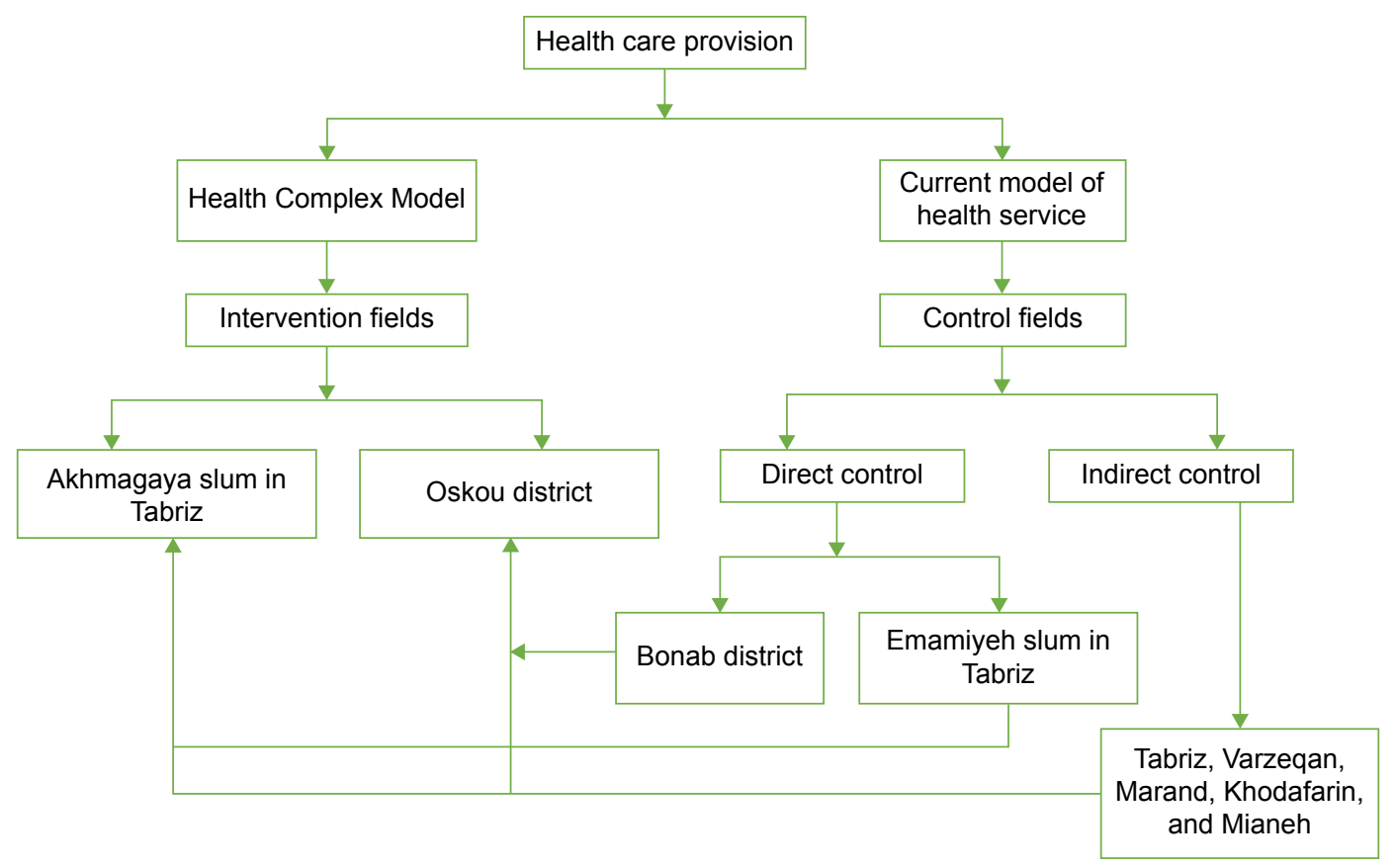

Figure I Methodology diagram of the research protocol to assess the effectiveness of the Health Complex Model in Iranian health reform.

complex centers and the population covered by them. The control group includes the health centers providing the routine health care services through the traditional Iranian health care system and the population covered by these health centers. The intervention arm of the study consists of two intervention fields. The first intervention region was Akhmagaya slum area with a marginalized population of 64,417 people located in the southwest of Tabriz metropolitan. The second intervention area is Oskou, a southern district in East Azerbaijan, with a population of 105,524 people. The control arm of the study consists of two types of control fields called direct control (directly paired with each intervention area) and indirect control (a control group representative for the whole province). A direct control field is selected for each of the two intervention fields according to the population criteria, health indicators, and socioeconomic status comparable with the intervention fields. Emamiyeh slum area with a population of 41,918 people located close to Akhmagaya slum area is selected as the direct control for the first intervention field. Bonab district, another southern district of the province with a population of 146,145 people is selected as the direct control for Oskou intervention area.

An indirect control group (consisting of five districts) is also defined to be studied. These five districts are selected on the basis of population criteria, distance from Tabriz, rural to urban population ratio, and human resource to population ratio. Tabriz is selected independently from other districts, and four other districts among other comparable 19 districts are randomly selected. Finally, Varzeqan, Marand, Khodafarin, and Mianeh along with Tabriz are selected as the indirect control group (Figure 1).

\section{The health complex structure}

Tabriz Health Complex (THC) project was designed in 2013 in the provincial health center of East Azerbaijan. The Health Complex is assumed to deliver integrated care services to people in a defined catchment area against identified per capita payment, under district health center policies and regulations. Each health complex covers a population of 40,000-120,000 in a defined geographic area (based on population distribution and their access to care) and includes a management center, several health centers, and one comprehensive health center (CHC). A thorough census is conducted on the covered population and all the relevant information are recorded. All the information obtained from the census are entered into the Iranian Health System Software (Ministry of Health, Tehran, Iran), and based on the public access, a family physician, and a family health expert defined as a health care team are appointed for every 4,000 people. Based on population density, $2-4$ health teams $(8,000-16,000$ people) are usually defined in each health center. For each health center, a dietitian, a mental health professional, and an occupational/environmental health expert are defined. One of the physicians of the health center is in charge of the 
center. Each health center in the defined geographical area offers the defined service package to the population covered by the responsible health complex (Figure 2).

The management center is actually the first level of management. Health experts (family and school health expert, diseases control expert, and occupational and environmental health expert) are stationed in the management center who work on the basis of the predefined tasks (eg, planning, training, research, monitoring, and evaluation) and are accountable for the health of the local population. Logistic and financial support experts are also located in the management center. $\mathrm{CHC}$ is one of the health centers that has the best suitable and accessible building among health centers to deliver integrated care services. Also, 24-hour emergency and ambulatory services and a referral clinic are located in this center. The referral clinic with at least four specialists (internist, pediatrician, gynecologist, and psychiatrist) accepts patients referred from health centers according to specified weekly schedule and prior arrangement while sending a relevant feedback to the general practitioner who has referred patients from the peripheral units to the referral clinic. In addition to accepting referrals, specialists are responsible for monitoring family physician referrals, follow-up of referred patients, training of family physicians, and responding to their telephone counseling. Nutrition and psychiatry consultation, dentistry services (free of charge identified services for children $<14$ years of age and pregnant women), and some social services are delivered in $\mathrm{CHC}$ as well. Health complexes can be administered as public, private, cooperative, or a combination of these entities. Details of the HCM are reported in a parallel report (Tabrizi, unpublished data, 2015). The intervention protocol is described in detail in a separate report.

\section{Survey outcome measures}

In order to assess the effectiveness of the intervention model, various outcomes are defined to be measured across several

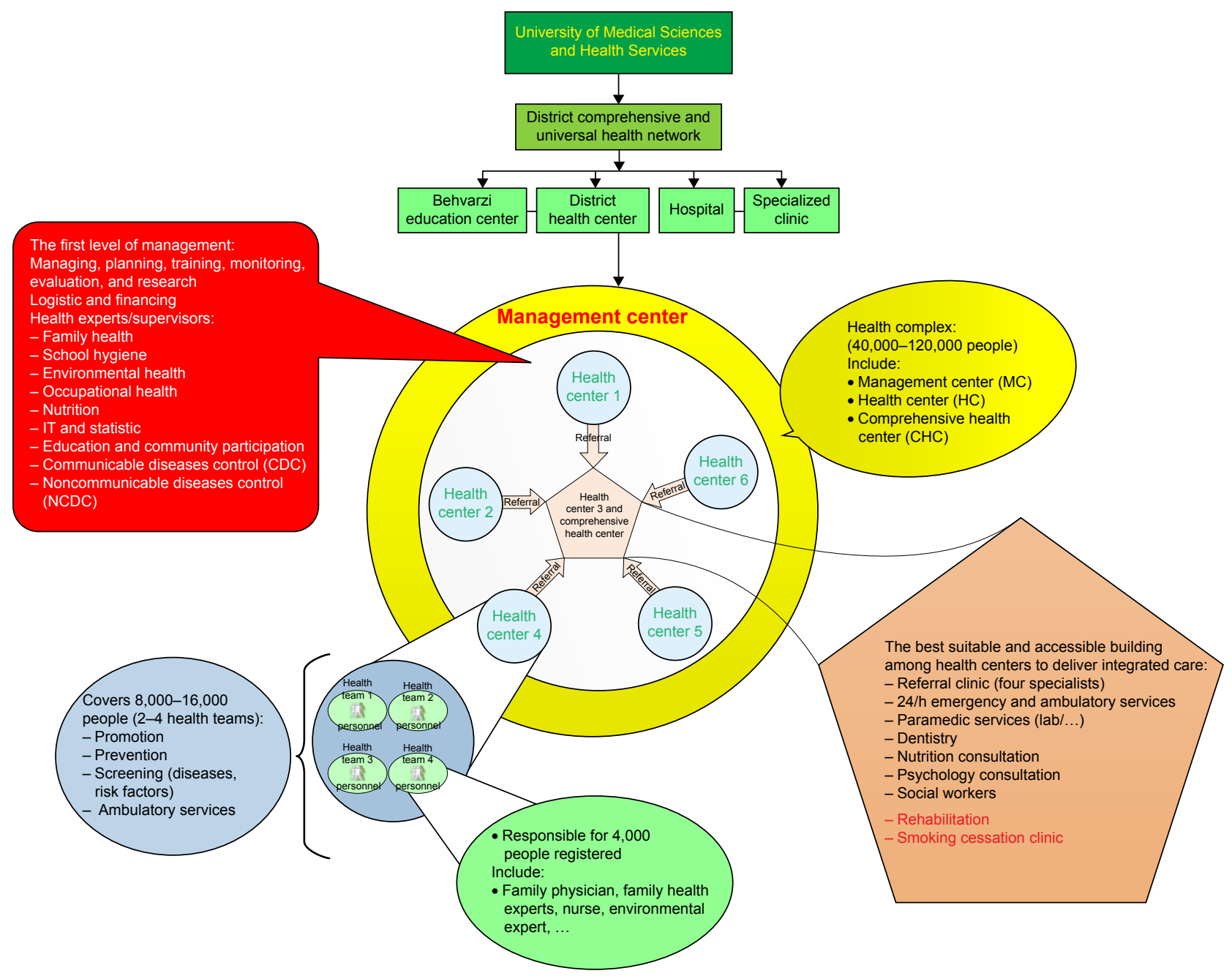

Figure 2 The preliminary Health Complex Structure in Iranian primary health care system.

Abbreviation: IT, information technology. 
sampling units in the present study and will be compared between intervention and control groups. The main outcomes in this regard include community empowerment and participation, PHC trust, national health policy trust, noncommunicable disease risk factors profile, self-care, health care utilization and responsiveness, maternity health care, child health care and vaccination, quality climate status in health facilities, diabetic care and management, hypertension care and management, depression care and management, acute care capacity, PHC data management status, and health equity. Various tools are being used from previous studies or are designed and validated through the project.

\section{Household questionnaire}

Household questionnaire includes questions about age, sex, literacy, job, and insurance situation of the family members. Questions about having pregnant woman, having child of $<6$ years of age, members with diabetes or hypertension or depression, traffic injured person, fallen or burned are also included. Access to the Internet and its usage rate in a day and week are asked from the households. This questionnaire is completed by one for each of the 6,000 households.

\section{Psychiatric assessment tools}

Psychiatric assessment tools include the following: attention deficit hyperactivity disorder tool: a standard questionnaire including 30 questions is used in the study which was validated in Persian by Sadeghi-Bazargani et al. ${ }^{11}$ Patient health questionnaire (PHQ): a validated questionnaire with 10 questions to screen depression. General health questionnaire (GHQ): a questionnaire with 28 questions validated in Persian by Noorbala. ${ }^{12}$ PHQ and GHQ questionnaires are completed by a single randomly selected adult living in households 11-12 in each cluster of 20 households.

\section{Pregnancy care questionnaire}

Pregnant women answer about their age, pregnancy situation (wanted or unwanted), care provider, health care services received, and their knowledge about the pregnancy risk factors. All pregnant women living in any of the 20 households in each cluster are enrolled.

\section{Nutrition status}

Frequency of the consumption of various food groups and food insecurity are assessed using a valid and reliable instrument. Validity and reliability of the original instrument in Persian are reported earlier. ${ }^{13}$ Information on food intake will be collected by using a 24-hour recall method for 3 days (one weekend day included). This is performed only in Osku intervention area and Bonab control area.

\section{Community empowerment and participation}

Health system performance in community empowerment and public participation in health care are surveyed. This questionnaire is completed by one for each of the households $1-5$ in every cluster.

\section{PHC trust}

This is a modified questionnaire from Public Trust on health care. It includes 31 questions regarding individuals' trust on PHC provided by health system. A national health policy trust is also developed. The psychometric properties of this scale will be published separately. The questionnaires are completed by a single randomly selected adult living in households $6-10$ in each cluster of 20 households.

\section{Risk factors questionnaire}

Modified Persian version of WHO STEPS questionnaire is used. Fast food consumption, smoking, narcotic consumption, and leisure time activities are explored using this instrument. ${ }^{14}$ This questionnaire is completed by one for a single randomly selected adult living in each of the five households (households 1-5) in every cluster.

\section{Self-care}

This questionnaire is developed by the researchers through the current research project. Its reliability and validity are confirmed and will be published separately. This questionnaire is completed by one for a single randomly selected adult living in each of the five households (households 1-5) in every cluster.

\section{Socioeconomic status}

SESIran is used to assess the socioeconomic status. It is a 22-item questionnaire in its full version developed for assessing socioeconomic status among Iranian households. The tool is available in full, short, and ultra-short versions. ${ }^{15,16}$ This questionnaire is completed by one for each of the 12 households (households 1-12) in every cluster.

\section{Health care utilization and responsiveness}

This includes 17 items about health services utilization and health system responsiveness validated earlier by Gharaie et al. ${ }^{17}$ This questionnaire is completed by one for each of the five households (households 6-10) in every cluster.

\section{Under 6-year-old child health care questionnaire}

This questionnaire asks about vaccination status, child care provider, child growth, and other factors in child care. It is 
completed by one for the children living in each of the 6,000 households.

\section{PHC data management questionnaire}

This questionnaire includes 20 questions to assess the health information process. It is completed by health workers and physicians, one randomly selected from each center.

\section{Modified Minnesota questionnaire}

It includes 20 items to assess job satisfaction ${ }^{18}$ and is completed by health workers and physicians, one randomly selected from each center.

\section{Quality climate scale}

It includes 42 items to assess quality climate at the organization and is completed by health workers and physicians, one randomly selected from each center.

\section{Other assessments}

These include currently available data through the national or provincial surveillance system on communicable diseases, noncommunicable diseases, environmental health, health education, and family health. The resting blood pressure and body mass index are also measured for adults $>17$ years of age. $\mathrm{HbA1c}$ will be assessed for 200 diabetic samples from intervention group and 200 samples from control group participants once at the end of the study.

\section{Sampling and data collection}

Household samples: three types of samples are enrolled in this study.

1. Population samples: two-stage cluster sampling method through probability proportional to size (PPS) routine is used to enroll population samples. At the first stage, total random sample of households are selected through cluster sampling method. A total of 2,400 households belong to the areas covered by HCM (new model). A total of 3,600 households belong to the control group, that is, the population covered by the traditional PHC system. At the second stage, individuals are sampled according to the various outcome measures described in detail earlier in this protocol under the section "Survey outcome measures".

2. Client samples: a total of 10 consecutive clients each day from among those referred to each of the health care units are enrolled over five week days.

3. Staff samples: one randomly selected physician and one randomly selected health care staff in each center/unit are interviewed.
The intervention group is composed of 1,200 households (ie, sixty clusters sized 20 households each) in each of the two intervention sites. Sixty clusters in Akhmaghia slum area, Tabriz, are selected on the basis of the census forms in 2014 using cluster sampling and also in Oskou district on the basis of the census records of urban and rural population using the same sampling methodology.

Clusters devoted to direct control groups are sixty 20-household clusters (1,200 households). Clusters are selected for the direct control group in the Emamieh area, Tabriz, according to family records under the coverage of the health center in the area. For Bonab (which is selected as the direct control for Oskou), clusters are selected on the basis of the census records of urban and rural populations using PPS cluster sampling.

The indirect control group consists of five cities representative of the province. The number of clusters selected is 60 clusters each including 20 households. The share of each city is determined on the basis of PPS. The clusters are selected on the basis of the census records of urban and rural population. For the Tabriz metropolitan, sixty 20-household clusters are separately allocated. In Tabriz metropolitan, clusters are selected on the basis of the framework of the Demographic Health Survey study (national survey in 2011). As collecting data for the large number of questions, through various data collection tools in this study, could affect the quality of collected information as well as the attrition rate, a specific plan is applied to allocate different data collection sets into five separate groups of households in each cluster (Table 1). This methodology although restricts the possibilities of making pairwise comparisons for all the scales used for data collection but, however, ensures the quality of data

Table I Data collection plan in each sampling cluster of the effectiveness research for Health Complex Model in Iran

\begin{tabular}{|c|c|c|}
\hline Number & Households & Questionnaire \\
\hline 1 & $\mathrm{I}-5$ & $\begin{array}{l}\text { Household information, community } \\
\text { empowerment and participation, } \\
\text { socioeconomic situation, risk factors } \\
\text { questionnaire, self-care }\end{array}$ \\
\hline 2 & $6-9$ & $\begin{array}{l}\text { Household information, PHC trust, } \\
\text { socioeconomic situation, health care } \\
\text { utilization, and responsiveness }\end{array}$ \\
\hline 3 & 10 & $\begin{array}{l}\text { Household information, socioeconomic } \\
\text { situation, } \mathrm{PHQ}, \mathrm{ADHD}, \mathrm{GHQ} \text {, health care } \\
\text { utilization and responsiveness, } \mathrm{PHC} \text { trust }\end{array}$ \\
\hline 4 & $11-12$ & $\begin{array}{l}\text { Household information, socioeconomic } \\
\text { situation, } \mathrm{PHQ}, \mathrm{ADHD}, \mathrm{GHQ}\end{array}$ \\
\hline 5 & $13-20$ & Household information \\
\hline
\end{tabular}

Abbreviations: $\mathrm{PHC}$, primary health care; $\mathrm{ADHD}$, attention deficit hyperactivity disorder; $\mathrm{PHQ}$, patient health questionnaire; GHQ, general health questionnaire. 
not to be affected because of overloading the participants with tremendous number of questions.

The direct interview method is used to collect information from households. For interviews, some interviewers are selected and trained during a meeting. For every five interviewers, for each team, a trained person is selected as the head of the team. In order to ensure the quality of data collection, $10 \%$ of all the completed daily household information is controlled by an external observer using telephone quality check interviews.

A pilot survey is conducted on 40 households prior to the main survey for 2 days in order to determine the data collection duration for each household interview, identify potential practical barriers, clarify and homogenize the data collection procedures, and improve family cooperation.

Interviews to collect household level information are mostly done with the family head or the most knowledgeable member of the family. The risk factor, self-care, attention deficit hyperactivity disorder, PHQ, and GHQ questionnaires and the trust questionnaire are completed for all $>18$-year individuals living in interviewed households defined separately for each instrument under the section on survey outcome measures in this article. People who have filled the self-care questionnaire are referred to the related health center to measure their height, weight, blood pressure, and waist circumference. These measurements are done by trained health care staff with at least 1 year of experience. Blood pressure is measured in the right arm with the subject in a supine position at resting status. A mercury-type sphygmomanometer is used to measure the blood pressure. Weight is measured with a Seca digital balance $(\mathrm{GmbH} \& \mathrm{Co}$, Hamburg, Germany) supplied by provincial health department. Height is measured with a wall-mounted stadiometer.

Each night all the completed questionnaires are checked for completeness, and the data are later entered into the computer using a specifically designed Microsoft Access database application (Microsoft Corporation, Redmond, WA, USA).

\section{Power calculation}

The overall sample size is determined to include 6,000 households with expected 24,000 household members. Considering the large number of measurements, this large sample size is decided to be included as the whole sample. To make comparisons on specific outcomes, the smallest comparison pair is assured to exist in a size of 344 in order to detect at least 0.15 difference in proportions with a maximum variance of $0.297\left(\mathrm{pq}=0.475^{*} 0.625\right)$ assuming type I random error to be at a maximum of 0.05 and type II random error to be at a maximum of 0.2. Multiple power calculations were made using Stata version 11 software package (StataCorp LP, College Station, TX, USA).

\section{Statistical methods}

According to the data scales, data distributions, and types of specific hypotheses for each subsample, a large variety of statistical methods may be applied to analyze the data; however, the main statistical methods that will be applied are summarized as follows.

\section{Descriptive statistics}

Mean and standard deviation, 95\% confidence intervals (CIs), median, and interquartile range will be reported for numeric scales. Proportions and their 95\% CIs will be reported for categorical measures.

\section{Bivariate associations}

According to the characteristics of the data and with respect to hypotheses, the chi-square test, Fishers' exact test, independent sample $t$-test, paired $t$-test, one-way analysis of variance, log-rank test, and appropriate nonparametric tests will be applied for bivariate analysis.

\section{Multivariate tests}

Logistic regression, linear regression, quintile regression, cox regression, and generalized linear models may be applied according to the characteristics of the data and the hypotheses.

\section{Ethical issues}

Study protocol is approved by the regional committee of ethics in Tabriz University of Medical Sciences. Written informed consent is obtained from all the participants of the study during the survey.

\section{Discussion}

In several countries, health system reform and interventions are frequently conducted at the PHC system in various ways, but in many cases, the effectiveness of them cannot be defended because the effectiveness is not evaluated through independent evaluation research projects or not using comprehensive and controlled research designs. Use of precise research methods with sufficient validity and reliability for national macroeconomic policy-making and decision-making on the cancellation, extension, or modification of these programs is necessary prior to implementing 
national-level health reforms. ${ }^{19-21}$ The present study has tried to gain its highest accuracy in assessments and a moderate comprehensiveness in the evaluation of the effectiveness due to the time and research financial restrictions. The research methodology reported in this article has considered few priorities in design of the evaluations including a priority on slum areas and some target groups, a priority on specific health problems, a priority on the development and use of valid instruments, and a priority on use of controlled methodology. Controlled trials either adaptive trials or the randomized trials are highly preferred to the easier-to-conduct before-after trials in controlling the external and confounding factors. ${ }^{22}$ Before and after studies, that is, quasi-experimental studies, are intrinsically weak to evaluate the effectiveness of health-related interventions in that secular trends, or sudden variations make it unreliable to attribute observed changes to the intervention of interest. On the other hand, conducting the controlled field trials is very expensive and sometimes practically impossible or not costeffective..$^{23,24}$

Design of the present study was a field trial with two types of control groups. One group is called the direct or paired control group. This helps to increase the baseline similarities between the intervention and control populations such that if the HCM as the intervention covers a slum area, the direct control group will also be a slum area with highest compatibility. However, to improve the interpretability of the results, the authors also used a representative sample of the whole province population called as indirect control. This helps to improve the statistical power either. Although, for practical reasons, it was not possible to design a randomized design instead of the adaptive design used in the present study, care was taken in selecting the control populations to the highest similarity as possible.

A characteristic of the present study is its moderate comprehensiveness. Actually, fully comprehensive studies may be impractical because of either cost or practical limitations; however, the present study seems acceptably comprehensive to judge on the overall effectiveness of the intervention while complementary assessments could also be considered to be added in future. This is while previous studies in assessing the effectiveness of new health policies have been either absent or much more limited with respect to the comprehensiveness or methodology. Nevertheless, communicable disease and health education are the two areas of limitation in the present study without specific survey-based assessments. Such areas will be compared only on the basis of the availability of information extracted from national surveillance data. Use of current statistics and surveillance data provides a more affordable way of collecting data along with the data collected through costly survey assessments that will also be performed on priority areas such as hypertension, diabetes, common psychiatric disorders, as well as child and maternity health care services in present studies. The present study has included statistics of performance including both the internal and the external clients of health system in order to depict a more detailed picture on how the health reforms react. This was used in previous studies on health cooperatives as public-private partnership model in Iran. ${ }^{9}$ The comprehensive research design being used in this study, although seems a bit expensive for a middle-income country, makes it reasonable for the granting body to support the study. ${ }^{25}$

The wide range of effectiveness outcomes to be measured in this study requires the researchers to use various multiple scales for measuring them. The first limitation with this is that increased number of questions to be asked at each household level may lead to some information bias. This is easy to manage by dividing the measurement instruments into five parts of households in each cluster. The second and main challenge is to find validated scales in Persian language for measuring the outcomes of interest. Some of the scales used in the present study are validated in previous studies; however, in some areas, no such tools are available in Persian. To overcome this, a budget is allocated for developing or adopting required validated scales through the standard psychometrics methodology as part of the research project. Few postgraduate thesis projects are also linked to facilitate the process. The results of the psychometric studies will be published separately.

Four noncommunicable diseases are considered for investigation in this study. Everyone knows about the priority of diabetes and hypertension to be thoroughly investigated in any health reform effectiveness study due to their prevalence and multiple interactive effects on human health as well as high costs of them on governments and common national wealth. Hospitalization due to diabetes is shown in a recent national study to have significant financial burden on individual, insurer, government, and the society in Iran. ${ }^{26}$ These are chronic conditions that if managed in proper way could largely improve the population health, and the PHC system plays an important role in this. ${ }^{26-31}$ The prevalence, severity, self-control, and related health care indices are being assessed in the present study through interviews, review of medical records, physical examination, and biological assays.

Depression is a major public health problem due to its high prevalence and affects health and quality of life. WHO 
estimates the depression to be the second cause of disability and burden of diseases in LMICs in $2020 .^{32,33}$ In the present study, depression is evaluated both for its prevalence and severity and for the care utilized in this respect.

Other than hypertension, diabetes, and psychiatric disorders, a limited assessment was also considered in the present evaluation research. Injuries especially the burns, traffic injuries, and falls are among the main health challenges in Iran as well as other developing countries. Approximately 1.27 million deaths occur each year and 20-50 million injuries are caused by traffic accidents; $90 \%$ of the whole share belongs to LMICs. Although injuries are quite complex multisectorial problem, any $\mathrm{PHC}$ reform program needs to consider reducing the burden of injuries by either primary or secondary and tertiary prevention strategies. $^{34}$

\section{Limitations and strengths Limitations}

1. Satisfaction with health care services is not surveyed specifically in this project. However, as trust is closely weaved with satisfaction, we believe that high trust could be an indicator of high satisfaction.

2. Due to budgeting restrictions, serum HbAlc is planned to be measured only 2 years after the intervention and no baseline will be assessed.

3. As like most nationally started health interventions, randomization is not possible in this study. This should be considered as a limitation of the study, but the methodology used in selecting the controls will be helpful to assess the reform compared to most other studies that have been conducted in a quasi-experimental design. Abundance of the outcomes is another aspect of the study that is considered to help in this regard through mixing the potential confounding effect.

4. With respect to age target groups, child and maternity health have been of focused investigations in present study; however, due to budgeting and practical limitations, no specifically focused investigation is planned for the elderly population.

5. This project is supported for a short-term evaluation and its extension to longer periods and a national-level extension may be required in order to judge the health reform model in a trustworthy way.

\section{Strengths}

1. A comprehensive study assessing most major fields of the effectiveness for health reform.
2. Use of previously validated data collection tools or development and validation of the study tools through standard psychometric methodology.

3. The large scale of study and adequately strict methodology ensuring the reliability and generalizability of results.

4. Limited number of questions to be answered at each session by each household through dividing the tools among five groups of households in each cluster.

\section{Acknowledgments}

The authors are thankful to Mr Saadati and Mrs Amanati for their help and comments throughout the work.

\section{Author contributions}

All authors contributed toward data analysis, drafting and critically revising the paper, gave final approval of the version to be published, and agree to be accountable for all aspects of the work.

\section{Disclosure}

The authors report no conflicts of interest in this work.

\section{References}

1. Boelen $\mathrm{C}$. The challenge of changing medical education and medical practice. World Health Forum. 1993;14(3):213.

2. Preker AS, Harding A, Travis P. "Make or buy" decisions in the production of health care goods and services: new insights from institutional economics and organizational theory. Bull WHO. 2000;78(6): 779-790.

3. Frenck J. The public/private mix and human resources for health. Health Policy Plann. 1993;8(4):315-326.

4. Pierre-Louis AM, Akala FA, Karam HS. Public health in the Middle East and North Africa: meeting the challenges of the twenty first century. The World Bank, Washington, DC; 2004.

5. King M. An Iranian experiment in primary health care: the West Azerbaijan project. Ormia Takvin. 2000;1379:1-7.

6. Shadpour K. Primary health care networks in the Islamic Republic of Iran. EMHJ. 2000;6(4):822-825.

7. Farzadfar F, Murray CJL, Gakidou E, et al. Effectiveness of diabetes and hypertension management by rural primary health-care workers (Behvarz workers) in Iran: a nationally representative observational study. Lancet. 2012;379(9810):47-54.

8. Malekafzali H. Primary health care in the rural area of the Islamic Republic of Iran. Iranian J Publ Health. 2009;38(1):69-70.

9. Farahbakhsh M, Sadeghi-Bazargani H, Nikniaz A, Tabrizi JS, Zakeri A, Azami S. Iran's experience of health cooperatives as a public-private partnership model in primary health care: a comparative study in East Azerbaijan. Health Promot Perspect. 2012;2(2):287-298.

10. Moradi-Lakeh M, Vosoogh-Moghaddam A. Health sector evolution plan in Iran; equity and sustainability concerns. Int $J$ Health Policy Manag. 2015;4(10):637-640.

11. Sadeghi-Bazargani H, Amiri S, Hamraz S, Malek A, Abdi S, Shahrokhi H. Validity and reliability of the Persian version of Conner's adult ADHD rating scales: observer and self-report screening versions. $J$ Clin Res Gov. 2014;3:42-47. 
12. Noorbala AA, Yazdi SAB, Mohammad K. The validation of general health questionnaire-28 as a psychiatric screening tool (text in Persian). Hakim Med J. 2009;11(4):47-53.

13. Mirmiran P, Hosseini F, Azizi F. Ravaei va Payaeiyeh Porseshnamehyeh Basamadeh Gazaei [Validity and reliability of the food frequency questionnaire]. Iranian J Diabet Lipid Disord. 2010;9(2):185197. Persian.

14. Sadeghi-Bazargani H, Jafarzadeh H, Fallah M, et al. Risk factor investigation for cardiovascular health through WHO STEPS approach in Ardabil, Iran. Vasc Health Risk Manag. 2011;7:417-424.

15. Aboubakri O, Sadeghi-Bazargani H, Asghari-Jafarabadi M, et al. Development and psychometric evaluation of a socioeconomic status questionnaire for urban households (SESIran): the primary version. Health Promot Perspect. 2016;5(4):250-260.

16. Sadeghi-Bazargani H, Aboubakri O, Asghari-Jafarabadi M, et al. Psychometric properties of the short and ultra-short versions of socioeconomic status assessment tool for health studies in Iran (SES-Iran). J Clin Res Gov. 2016;5(1).

17. Gharaie H, Bahrami MA, Hamidi Y, Askari R, Tapak L. The relationship between organizational commitment and accountability in the public, private and charitable hospitals in the city of Hamedan and Yazd in 1392. J Health Manag. 2013;3(1-2):35-47.

18. Gholami Fesharaki M, Talebian D, Aghamiri Z, Mohamadian M. Reliability and validity assessments for Najmie job satisfaction questionnaire. Arak Univ Med Sci J. 2011;14(5):63-69.

19. Larizgoitia I, Starfield B. Reform of primary health care: the case of Spain. Health Policy. 1997;41(2):121-137.

20. Brownell MD, Roos NP, Roos LL. Monitoring health reform: a report card approach. Soc Sci Med. 2001;52(5):657-670.

21. Gakidou E, Lozano R, González-Pier E, et al. Assessing the effect of the 2001-2006 Mexican health reform: an interim report card. Lancet. 2006;368(9550):1920-1935.

22. Shapiro HKAS. Epidemiligy and Health Services. New York: Oxford University Press; 1998.
23. Grimshaw J, Campbell M, Eccles M, Steen N. Experimental and quasi-experimental designs for evaluating guideline implementation strategies. Fam Pract. 2000;17(Suppl 1):S11-S16.

24. Eccles M, Grimshaw J, Campbell M, Ramsay C. Research designs for studies evaluating the effectiveness of change and improvement strategies. Qual Saf Health Care. 2003;12(1):47-52.

25. Mehrdad R. Health system in Iran. JMAJ. 2009;52(1):69-73.

26. Ghaffari S, Hashemi SE, Atabaki H. The national financial burden of hospitalization of diabetes in Iran. J Clin Res Gov. 2012;1(1):16-21.

27. Ahmadvand AR, Jamshidy HR, Sotodeh M, et al. World Health Report 2002: Reducing Risks, Promoting Healthy Life. Tehran: Ibn Sina Institute Great (World Health Organization); 2002.

28. Staessen JA, Gasowski J, Wang JG, et al. Risks of untreated and treated isolated systolic hypertension in the elderly: meta-analysis of outcome trials. Lancet. 2000;355(9207):865-872.

29. Roglic G, Unwin N, Bennett PH, et al. The burden of mortality attributable to diabetes: realistic estimates for the year 2000. Diabetes Care. 2005;28(9):2130-2135.

30. Al-Dossary RN, Kitsantas P. The impact of diabetes nurse case management on hemoglobin A1c (HgbA1c) and self-efficacy of patients with type 2 diabetes: a systematic review. J Clin Res Gov. 2014;3(1): 9-15.

31. Funnell MM, Anderson RM. Empowerment and self-management of diabetes. Clin Diabetes. 2004;22(3):123-127.

32. Ozdemir H, Rezaki M. Depresyon Saptanmasında Genel Sağlık Anketi-12 [General Health Questionnaire-12 for the detection of depression]. Turk Psikiyatri Derg. 2007;18(1):13-21. Turkish.

33. Noble RE. Depression in women. Metabolism. 2005;54(5 Suppl):49-52.

34. World Health Organization. Global status report on road safety 2013. Available from: http://www.who.int/violence_injury_prevention/ road_safety_status/2013/en/. Accessed June 10, 2016.
Patient Preference and Adherence

\section{Publish your work in this journal}

Patient Preference and Adherence is an international, peer-reviewed, open access journal that focuses on the growing importance of patient preference and adherence throughout the therapeutic continuum. Patient satisfaction, acceptability, quality of life, compliance, persistence and their role in developing new therapeutic modalities and compounds to optimize

\section{Dovepress}

clinical outcomes for existing disease states are major areas of interest for the journal. This journal has been accepted for indexing on PubMed Central. The manuscript management system is completely online and includes a very quick and fair peer-review system, which is all easy to use. Visit http://www. dovepress.com/testimonials.php to read real quotes from published authors. 